Proyecciones Journal of Mathematics

Vol. 35, $\mathrm{N}^{o}$ 1, pp. 41-61, March 2016.

Universidad Católica del Norte

Antofagasta - Chile

\title{
Sufficient conditions for the boundedness and square integrability of solutions of fourth-order differential equations
}

\author{
Moussadek Remili \\ University of Oran, Algeria \\ and \\ Mebrouk Rahmane \\ University of Oran 1, Algeria \\ Received: March 2015. Accepted: November 2015
}

\begin{abstract}
Sufficient conditions for the boundedness and square integrability of solutions and their derivatives of certain fourth order nonlinear differential equation are given by means of the Lyapunov's second method. Our results obtained in this work, generalize existing results on fourth order nonlinear differential equations in the literature. For illustration, an example is also given.
\end{abstract}

2010 Mathematics Subject Classification : $34 C 11$.

Keywords and phrases : Boundedness, stability, Lyapunov functional, fourth-order differential equations, square integrable. 


\section{Introduction}

During the past few years there has been many excellent results concerning the boundedness of the solutions of nonlinear ordinary differential equations by the use of the Lyapunov's method (Yoshizawa [27]). Today, this method is widely recognized as an excellent tool not only in the study of differential equations but also in the theory of control systems, dynamical systems, systems with time lag, power system analysis, time varying nonlinear feedback systems, and so on. But, finding an appropriate Lyapunov function for higher order differential equations is in general a difficult task. Many works concerning have investigated the boundedness of solutions of certain differential equations of the fourth order. We mention, the works of Ezeilo [6], [7], Harrow [8], [9], Afuwape and Adesina [1], Tiryaki and Tunç [17], Tunç [18], [19], [20], Tunç and Tiryaki [21] where the Lyapunov's second method was used.

In [12], Omeike by using the Cauchy formula for the particular solution of nonlinear differential equations with constant coefficients, has proved that the solution of the equation

$$
x^{\prime \prime \prime \prime}+a x^{\prime \prime \prime}+b x^{\prime \prime}+c x^{\prime}+h(x)=p(t)
$$

and its derivatives up to order three are bounded.

In [22], and [25] Tunç established sufficient conditions for the asymptotic stability of the zero solution and the boundedness of the the following equations

$$
\begin{aligned}
x^{\prime \prime \prime \prime}+a_{1} x^{\prime \prime \prime}+\psi\left(x, x^{\prime}\right) x^{\prime \prime}+a_{4} x^{\prime}+h(x) & =0, \\
x^{\prime \prime \prime \prime}+a_{1} x^{\prime \prime \prime}+\psi\left(x, x^{\prime}\right) x^{\prime \prime}+g\left(x^{\prime}\right)+a_{4} x & =0, \\
x^{\prime \prime \prime \prime \prime}+a x^{\prime \prime \prime}+\psi\left(x, x^{\prime}, x^{\prime \prime}\right)+g\left(x, x^{\prime}\right)+h(x) & =p(t) .
\end{aligned}
$$

The problem related to the study of square integrability of solutions for higher order nonlinear differential equations is also of great interest, but it should be noted that only a few results are related to the fourth order nonlinear differential equations. In 1989 Andres and Vlček [2], discussed the square integrable solutions of (1.1). 
The purpose of this paper is to define a Lyapunov function and use it to study the boundedness of solutions and we also study the square integrability of solutions of the differential equation of the form

$$
\begin{aligned}
& \quad\left(g(x(t)) x^{\prime \prime}(t)\right)^{\prime \prime}+a(t)\left(p(x(t)) x^{\prime \prime}(t)\right)^{\prime}+b(t)\left(q(x(t)) x^{\prime}(t)\right)^{\prime} \\
& +\mathrm{c}(t) f(x(t)) x^{\prime}(t)+d(t) h(x(t))=e(t),
\end{aligned}
$$

where $a(t), b(t), c(t), d(t), e(t), f(x), g(x), p(x), q(x)$ and $h(x)$ are continuous functions depending only on the arguments shown and $p^{\prime}(x), q^{\prime}(x), f^{\prime}(x)$ and $h^{\prime}(x)$ exist and are continuous.

Hence, the aim of this paper consists mainly in further extension of the related existing results.

\section{Assumptions and main results}

We begin by presenting some sufficient assumptions which will be used in equation (1.5), and suppose that there are positive constants

$a_{0}, b_{0}, c_{0}, d_{0}, f_{0}, g_{0}, p_{0}, q_{0}, a_{1}, b_{1}, c_{1}, d_{1}, f_{1}, g_{1}, p_{1}, q_{1}, m, M, \delta, \eta_{1}, h_{0}, \delta_{0}$, such that the following conditions are satisfied

i) $0<a_{0} \leq a(t) \leq a_{1} ; 0<b_{0} \leq b(t) \leq b_{1} ; \quad 0<c_{0} \leq c(t) \leq c_{1} ; 0<$ $d_{0} \leq d(t) \leq d_{1}$ for $t \geq 0$.

ii) $0<f_{0} \leq f(x) \leq f_{1} ; g_{0} \leq g(x) \leq g_{1} ; 0<p_{0} \leq p(x) \leq p_{1} ;$ $0<q_{0} \leq q(x) \leq q_{1}$ for $x \in R$ and $0<m<\min \left\{f_{0}, p_{0}, g_{0}, 1\right\}, M>\max \left\{f_{1}, g_{1}, p_{1}, 1\right\}$.

iii) $\quad \frac{h(x)}{x} \geq \delta>0 \quad($ for $x \neq 0) ; h(0)=0$.

iv) $\frac{h_{0}}{m}-\frac{a_{0} m \delta_{0}}{d_{1}} \leq h^{\prime}(x) \leq \frac{h_{0}}{2 M} \quad$ for $\quad x \in R$. 
The following lemma will be needed in the proof of our results. [11] Let $h(0)=0, x h(x)>0(x \neq 0)$ and $\delta(t)-h^{\prime}(x) \geq 0(\delta(t)>0)$, then

$$
2 \delta(t) H(x) \geq h^{2}(x) \quad \text { where } \quad H(x)=\int_{0}^{x} h(s) d s
$$

Before stating the theorem, we introduce the following notations:

$$
\left\{\begin{array}{l}
\kappa_{1}=\frac{a_{1} h_{0} d_{1} M^{2}}{c_{0} m^{3}}+\frac{M^{3}\left(c_{1}+\delta_{0}\right)}{a_{0} m^{2}}+a_{0} a_{1} m(M-1), \\
\kappa_{2}=\frac{2 d_{1} h_{0} a_{0}}{c_{0}(M-1)}\left(\frac{1}{m}-\frac{1}{M}\right)^{2}+2 \frac{c_{0} M}{a_{0}}+2 a_{1} \frac{d_{1} h_{0} M}{c_{0} m^{3}}+\frac{c_{0} c_{1}\left(M^{2}+2\right) m M}{d_{1} h_{0}} .
\end{array}\right.
$$

In addition to conditions (i) (iv) being satisfied, assume that there are positive constants $\eta_{2}, \eta_{3}$ and $\eta_{4}$ such that the following conditions hold

$$
\begin{array}{ll}
\left.H_{0}\right) \quad b_{0} q_{0}>\max \left\{\kappa_{1}, \kappa_{2}\right\} . \\
\left.H_{1}\right) \quad \int_{0}^{+\infty}\left(\left|a^{\prime}(t)\right|+\left|b^{\prime}(t)\right|+\left|c^{\prime}(t)\right|+\left|d^{\prime}(t)\right|\right) d t<\eta_{1} . \\
\left.H_{2}\right) \quad \int_{-\infty}^{+\infty}\left(\left|g^{\prime}(s)\right|+\left|p^{\prime}(s)\right|+\left|q^{\prime}(s)\right|+\left|f^{\prime}(s)\right|\right) d s<\eta_{2} . \\
\left.H_{3}\right) \quad \int_{0}^{+\infty}|e(s)| d s<\eta_{3} . \\
\left.H_{4}\right) \quad\left|g^{\prime}(x)\right|<\eta_{4}, \text { for all } x .
\end{array}
$$

Then any solution $x(t)$ of (??) and its derivatives $x^{\prime}(t), x^{\prime \prime}(t)$ and $x^{\prime \prime \prime}(t)$ are bounded and satisfy

$$
\int_{0}^{\infty}\left(x^{2}(s)+x^{\prime 2}(s)+x^{\prime \prime 2}(s)+x^{\prime \prime \prime 2}(s)\right) d s<\infty .
$$

Proof. The equation (1.5) can be expressed as the following system 


$$
\left\{\begin{array}{l}
x^{\prime}=y, \\
y^{\prime}=\frac{1}{g(x)} z, \\
z^{\prime}=w, \\
w^{\prime}=-a(t) \frac{p(x)}{g(x)} w+\left(a(t) p(x) \theta_{1}-b(t) \frac{q(x)}{g(x)}-a(t) g(x) \theta_{2}\right) z \\
\quad \quad\left(b(t) g^{2}(x) \theta_{3}+c(t) f(x)\right) y-d(t) h(x)+e(t),
\end{array}\right.
$$

where

$$
\begin{aligned}
\theta_{1}(t)=\frac{g^{\prime}(x(t))}{g^{2}(x(t))} x^{\prime}(t), \theta_{2}(t) & =\frac{p^{\prime}(x(t))}{g^{2}(x(t))} x^{\prime}(t), \theta_{3}(t)=\frac{q^{\prime}(x(t))}{g^{2}(x(t))} x^{\prime}(t), \\
\theta_{4}(t) & =\frac{f^{\prime}(x(t))}{g^{2}(x(t))} x^{\prime}(t) .
\end{aligned}
$$

Boundedness of solutions: First we proof the boundedness of solutions. The proof depend on the Lyapunov function $W=W(t, x, y, z, w)$ defined as

$$
W=e^{-\frac{1}{\eta} \int_{0}^{t}\left(\gamma_{1}(s)+\gamma_{2}(s)\right) d s} V
$$

where

$$
\begin{aligned}
& \gamma_{1}(t)=\left|a^{\prime}(t)\right|+\left|b^{\prime}(t)\right|+\left|c^{\prime}(t)\right|+\left|d^{\prime}(t)\right|, \quad \gamma_{2}(t)=\left|\theta_{1}(t)\right|+\left|\theta_{2}(t)\right|+ \\
& \left|\theta_{3}(t)\right|+\left|\theta_{4}(t)\right|
\end{aligned}
$$

and

$$
\begin{aligned}
2 V & =2 V(t, x, y, z, w)=2 \beta d(t) H(x)+c(t) g(x) f(x) y^{2}+\alpha b(t) \frac{q(x)}{g(x)} z^{2} \\
& +\mathrm{a}(t) \frac{p(x)}{g(x)} z^{2}+2 \beta a(t) \frac{p(x)}{g(x)} y z+\left[\beta b(t) q(x)-\alpha h_{0} d(t)\right] y^{2}-\beta \frac{1}{g(x)} z^{2} \\
& +\alpha w^{2}+2 d(t) g(x) h(x) y+2 \alpha d(t) h(x) z+2 \alpha c(t) f(x) y z+2 \beta y w+
\end{aligned}
$$
$2 z w$,

with $\alpha=\frac{M}{a_{0} m}+\epsilon, \beta=\frac{d_{1} h_{0}}{c_{0} m}+\epsilon . \quad \epsilon, \eta$ are positive constants to be determined later in the proof. $2 V$ can be rearranged in the form

$$
2 V=a(t) p(x)\left(\frac{w}{a(t) p(x)}+z+\beta \frac{1}{g(x)} y\right)^{2}+c(t) f(x)\left(\frac{d(t) h(x)}{c(t) f(x)}+y+\alpha z\right)^{2}
$$


such that

$$
+\mathrm{c}(t) f(x)\left[(g(x)-1) y+\frac{d(t) h(x)}{c(t) f(x)}\right]^{2}+2 \epsilon d(t) H(x)+V_{1}+V_{2}+V_{3},
$$

$$
\begin{aligned}
& \mathrm{V}_{1}=2 d(t) \int_{0}^{x} h(s)\left(\frac{d_{1} h_{0}}{c_{0} m}-2 \frac{d(t)}{c(t) f(x)} h^{\prime}(s)\right) d s \\
& \mathrm{~V}_{2}=\left(\alpha b(t) \frac{q(x)}{g(x)}-\beta \frac{1}{g(x)}-\alpha^{2} c(t) f(x)+a(t) p(x)\left(\frac{1}{g(x)}-1\right)\right) z^{2}
\end{aligned}
$$

and

$$
\begin{gathered}
\mathrm{V}_{3}=\left(\beta b(t) q(x)-\alpha h_{0} d(t)-\beta^{2} a(t) \frac{p(x)}{g^{2}(x)}-c(t) f(x)\left(g^{2}(x)-3 g(x)+2\right)\right) y^{2} \\
+\left(\alpha-\frac{1}{a(t) p(x)}\right) w^{2}+2 \beta\left(1-\frac{1}{g(x)}\right) y w .
\end{gathered}
$$

Now we will prove that $V$ is positive definite. Take

$$
\epsilon<\min \left\{\frac{M}{a_{0} m}, \frac{d_{1} h_{0}}{c_{0} m}, \frac{m^{2}\left(b_{0} q_{0}-\kappa_{1}\right)}{M^{2}\left(a_{1}+m M c_{1}\right)}\right\},
$$

then

$$
\frac{M}{a_{0} m}<\alpha<2 \frac{M}{a_{0} m}, \quad \frac{d_{1} h_{0}}{c_{0} m}<\beta<2 \frac{d_{1} h_{0}}{c_{0} m} .
$$

Conditions (i) $\sim\left(\right.$ iv) and $\left(H_{0}\right)$ imply that

$$
V_{1} \geq 4 d_{0} \frac{d_{1}}{c_{0} m} \int_{0}^{x} h(s)\left(\frac{h_{0}}{2 M}-h^{\prime}(s)\right) d s \geq 0 .
$$

We can rewrite $V_{2}$ as

$$
\begin{aligned}
& \quad \mathrm{V}_{2}=\alpha\left(b(t) \frac{q(x)}{g(x)}-\beta \frac{a(t)}{g(x)}-\alpha c(t) f(x)-\frac{a(t) p(x)}{\alpha}\left(1-\frac{1}{g(x)}\right)\right) z^{2} \\
& +\beta\left(\alpha \frac{a(t)}{g(x)}-\frac{1}{g(x)}\right) z^{2} .
\end{aligned}
$$

From conditions (i) $\sim($ iii) and inequalities (2.3),(2.4), it follows that

$$
\begin{aligned}
& \mathrm{V}_{2} \geq \alpha\left(\frac{b_{0} q_{0}}{M}-\left(\frac{d_{1} h_{0}}{c_{0} m}+\epsilon\right) \frac{a_{1}}{m}-\left(\frac{M}{a_{0} m}+\epsilon\right) c_{1} M-a_{1} \frac{a_{0} m}{M}(M-1)\right) z^{2} \\
& +\beta\left(\alpha \frac{a_{0}}{M}-\frac{1}{m}\right) z^{2}
\end{aligned}
$$




$$
\begin{aligned}
& \geq \alpha\left(\frac{b_{0} q_{0}}{M}-\frac{d_{1} h_{0} a_{1}}{c_{0} m^{2}}-\frac{c_{1} M^{2}}{a_{0} m}-a_{1} \frac{a_{0} m}{M}(M-1)-\frac{\epsilon}{m}\left(a_{1}+c_{1} m M\right)\right) z^{2} \\
\geq & \frac{\alpha}{M m}\left(m\left(b_{0} q_{0}-\kappa_{1}\right)-\epsilon M\left(a_{1}+c_{1} m M\right)\right) z^{2} \geq 0,
\end{aligned}
$$

and

$$
\begin{aligned}
V_{3} \geq & \beta\left(b_{0} q_{0}-\frac{\alpha}{\beta} h_{0} d_{1}-a_{1} \beta \frac{M}{g^{2}(x)}-\frac{c_{1} M\left(M^{2}+2\right)}{\beta}\right) y^{2}+\left(\frac{M-1}{a_{0} m}\right) w^{2} \\
& +2 \beta\left(1-\frac{1}{g(x)}\right) y w \\
\geq & \beta\left(b_{0} q_{0}-2 \frac{M}{a_{0}} c_{0}-2 a_{1} \frac{d_{1} h_{0} M}{c_{0} m^{3}}-\frac{c_{0} c_{1}\left(M^{2}+2\right) m M}{d_{1} h_{0}}\right) y^{2}+\left(\frac{M-1}{a_{0} m}\right) w^{2} \\
& +2 \beta\left(1-\frac{1}{g(x)}\right) y w \\
\geq & \psi(y, \omega),
\end{aligned}
$$

such that

$\psi(y, \omega)=\beta \frac{2 d_{1} h_{0} a_{0}}{c_{0}(M-1)}\left(\frac{1}{m}-\frac{1}{M}\right)^{2} y^{2}+\left(\frac{M-1}{a_{0} m}\right) w^{2}+2 \beta\left(1-\frac{1}{g(x)}\right) y w$.

It is clear that $\psi(y, \omega)$ is positive definite. To show this we calculate the discriminant

$$
\triangle=\beta^{2}\left[1-\frac{1}{g(x)}\right]^{2}-\frac{2 \beta d_{1} h_{0}}{c_{0} m}\left[\frac{1}{M}-\frac{1}{m}\right]^{2} .
$$

Using condition (ii) we have

$$
\frac{1}{M}<\frac{1}{g(x)}<\frac{1}{m}, \text { and } \frac{1}{M}<1<\frac{1}{m}
$$

it follows that

$$
\left|1-\frac{1}{g(x)}\right|<\frac{1}{m}-\frac{1}{M}
$$

Using (2.4) we get

$$
\triangle \leq \beta\left[\frac{2 d_{1} h_{0}}{c_{0} m}\left(\frac{1}{M}-\frac{1}{m}\right)^{2}-\frac{2 d_{1} h_{0}}{c_{0} m}\left(\frac{1}{M}-\frac{1}{m}\right)^{2}\right]=0 .
$$


Thus there exists positive number $D_{0}$ such that

$$
2 V \geq D_{0}\left(y^{2}+z^{2}+w^{2}+H(x)\right) .
$$

By Lemma 2 and conditions (iii) and $\left(H_{1}\right)$ we get the existence of a positive number $D_{1}$ such that

$$
2 V \geq D_{1}\left(x^{2}+y^{2}+z^{2}+w^{2}\right),
$$

thus $V$ is positive definite which implies that $W$ is also positive definite.

Hence we can find positive definite functions $U_{1}(\|)$ and $U_{2}(\|X\|)(X=$ $(x, y, z, w))$ such that $U_{1}(\|X\|) \leq V \leq U_{2}(\|X\|)$. By (ii) and $\left(H_{2}\right)$, we get

$$
\begin{aligned}
& \int_{0}^{t}\left(\gamma_{1}(s)+\gamma_{2}(s)\right) d s \leq \eta_{1}+\int_{\alpha_{1}(t)}^{\alpha_{2}(t)} \frac{\left|g^{\prime}(u)\right|+\left|p^{\prime}(u)\right|+\left|q^{\prime}(u)\right|+\left|f^{\prime}(u)\right|}{g^{2}(u)} d u \\
& \leq \eta_{1}+\frac{1}{m^{2}} \int_{-\infty}^{+\infty}\left(\left|g^{\prime}(s)\right|+\left|p^{\prime}(s)\right|+\left|q^{\prime}(s)\right|+\left|f^{\prime}(s)\right|\right) d s \\
& \leq \eta_{1}+\frac{\eta_{2}}{m^{2}}
\end{aligned}
$$

with $\alpha_{1}(t)=\min \{x(0), x(t)\}$, and $\alpha_{2}(t)=\max \{x(0), x(t)\}$. By condition $\left(H_{1}\right)$ and inequalities $(2.2),(2.6)$ and $(2)$ we have

$$
W \geq D_{2}\left(x^{2}+y^{2}+z^{2}+w^{2}\right),
$$

where $D_{2}=\frac{D_{1}}{2} e^{-\frac{1}{\eta}\left(\eta_{1}+\frac{\eta_{2}}{m^{2}}\right)}$. Therefore we can find positive definite functions $W_{1}(\|X\|)$ and $W_{2}(\|X\|)$ such that $W_{1}(\|X\|) \leq W \leq W_{2}(\|X\|)$.

Next we show that $\dot{W}$ is negative definite functional. The derivative of the function $\mathrm{V}$, along any solution $(x(t), y(t), z(t), w(t))$ of system $(2.1)$, with respect to $t$ is after elementary calculation

$2 . V_{(2.1)}=-2 \epsilon c(t) f(x) y^{2}+V_{4}+V_{5}+V_{6}+V_{7}+2(\beta y+z+\alpha w) e(t)+2 \frac{\partial V}{\partial t}$,

where 


$$
\begin{aligned}
V_{4} & =-2\left(\frac{d_{1} h_{0}}{c_{0} m} c(t) f(x)-d(t) g(x) h^{\prime}(x)\right) y^{2}-2 \alpha d(t)\left(\frac{h_{0}}{g(x)}-h^{\prime}(x)\right) y z \\
\mathrm{~V}_{5} & =-2\left(\frac{b(t) q(x)}{g(x)}-\alpha c(t) \frac{f(x)}{g(x)}-\beta a(t) \frac{p(x)}{g^{2}(x)}\right) z^{2} \\
\mathrm{~V}_{6} & =-2\left(\alpha \frac{a(t) p(x)}{g(x)}-1\right) w^{2}
\end{aligned}
$$

and

$$
\begin{aligned}
V_{7}= & \theta_{1}\left(a(t) p(x) z^{2}-\alpha b(t) q(x) z^{2}+c(t) f(x) g^{2}(x) y^{2}+\beta z^{2}+2 d(t) g^{2}(x) h(x) y\right. \\
& +2 \alpha a(t) p(x) z w)-b(t) \theta_{3} g(x)\left(\alpha z^{2}+2 \alpha g(x) z w+\beta g(x) y^{2}+2 g(x) y z\right) \\
& -a(t) \theta_{2} g(x)\left(z^{2}+2 \alpha z w\right)+\theta_{4}\left(c(t) g^{3}(x) y^{2}+2 \alpha c(t) g^{2}(x) y z\right)
\end{aligned}
$$

By conditions (i), (ii), (iv), $\left(H_{0}\right)$ and inequalities $(2.3),(2.4)$ we get

$$
\begin{aligned}
V_{4} & \leq-2\left(d(t) h_{0}-d(t) g(x) h^{\prime}(x)\right) y^{2}-2 \alpha d(t)\left(\frac{h_{0}}{g(x)}-h^{\prime}(x)\right) y z \\
& \leq-2 d(t) m\left(\frac{h_{0}}{g(x)}-h^{\prime}(x)\right)\left[\left(y+\frac{\alpha}{2 m} z\right)^{2}-\left(\frac{\alpha}{2 m} z\right)^{2}\right] \\
& \leq \frac{\alpha^{2}}{2 m} d(t)\left(\frac{h_{0}}{m}-h^{\prime}(x)\right) z^{2} .
\end{aligned}
$$

Therefore,

$$
\begin{aligned}
& V_{4}+V_{5} \leq-2\left[\frac{b_{0} q_{0}}{M}-\left(\frac{M}{a_{0} m}+\epsilon\right) \frac{c_{1} M}{m}-\left(\frac{d_{1} h_{0}}{c_{0} m}+\epsilon\right) \frac{a_{1} M}{m^{2}}-\frac{\alpha^{2}}{4 m}\left(a_{0} \delta_{0}\right)\right] z^{2} \\
\leq & -2\left[\frac{b_{0} q_{0}}{M}-\frac{M^{2}}{a_{0} m^{2}} c_{1}-\frac{d_{1} h_{0} a_{1} M}{c_{0} m^{3}}-\frac{M^{2} \delta_{0}}{a_{0} m^{2}}-\epsilon \frac{M}{m}\left(\frac{a_{1}}{m}+c_{1}\right)\right] z^{2} \\
\leq & -\frac{2}{M m^{2}}\left(m^{2}\left(b_{0} q_{0}-\kappa_{1}\right)-\epsilon M^{2}\left(a_{1}+c_{1} m\right)\right) z^{2} \leq 0 .
\end{aligned}
$$

We have also,

$$
V_{6} \leq-2\left(\alpha \frac{a_{0} m}{M}-1\right) w^{2}=-2 \epsilon \frac{a_{0} m}{M} w^{2} \leq 0 .
$$


Putting

$$
D_{3}=\min \left\{\epsilon c_{0} m, \epsilon \frac{a_{0} m}{M}, \frac{1}{M m^{2}}\left(m^{2}\left(b_{0} q_{0}-\kappa_{1}\right)-\epsilon M^{2}\left(a_{1}+c_{1} m M\right)\right)\right\}
$$

we obtain

$$
-2 \epsilon c(t) f(x) y^{2}+V_{4}+V_{5}+V_{6} \leq-2 D_{3}\left(y^{2}+z^{2}+w^{2}\right) .
$$

From Lemma (2.1) and inequalities (2.5), $2 u v \leq u^{2}+v^{2}$ we obtain the following

$$
\begin{aligned}
& \mathrm{V}_{7} \leq\left|\theta_{1}\right|\left(a(t) p(x) z^{2}+\alpha b(t) q(x) z^{2}+c(t) f(x) g^{2}(x) y^{2}+\beta z^{2}+d(t) g^{2}(x)\right. \\
& \left(h^{2}(x)+y^{2}\right) \\
+ & \left.\alpha a(t) p(x)\left(z^{2}+w^{2}\right)\right)+\left|\theta_{4}\right|\left(c(t) g^{3}(x) y^{2}+\alpha c(t) g^{2}(x)\left(y^{2}+z^{2}\right)\right) \\
+ & b(t)\left|\theta_{3}\right| g(x)\left(\alpha z^{2}+\alpha g(x)\left(z^{2}+w^{2}\right)+\beta g(x) y^{2}+g(x)\left(y^{2}+z^{2}\right)\right) \\
+ & a(t)\left|\theta_{2}\right| g(x)\left(z^{2}+\alpha\left(z^{2}+w^{2}\right)\right) \\
\leq & \frac{2 K_{1}}{D_{0}}\left(\left|\theta_{1}\right|+\left|\theta_{2}\right|+\left|\theta_{3}\right|+\left|\theta_{4}\right|\right) V, .
\end{aligned}
$$

with $K_{1}$ some positive constant. We get also,

$$
\begin{aligned}
& 2 \frac{\partial V}{\partial t}= d^{\prime}(t)\left[2 \beta H(x)-\alpha h_{0} y^{2}+2 g(x) h(x) y+2 \alpha h(x) z\right] \\
&+c^{\prime}(t)\left[g(x) f(x) y^{2}+2 \alpha f(x) y z\right]+b^{\prime}(t)\left[\alpha \frac{q(x)}{g(x)} z^{2}+\beta q(x) y^{2}\right] \\
&+a^{\prime}(t)\left[\frac{p(x)}{g(x)} z^{2}+2 \beta \frac{p(x)}{g(x)} y z\right] \\
& \leq 2 \frac{K_{2}}{D_{0}}\left(\left|a^{\prime}(t)\right|+\left|b^{\prime}(t)\right|+\left|c^{\prime}(t)\right|+\left|d^{\prime}(t)\right|\right) V,
\end{aligned}
$$

with $K_{2}$ positive constant. Thus for $\frac{1}{\eta}=\frac{1}{D_{0}} \max \left\{K_{1}, K_{2}\right\}$ we have

$$
. V_{(2.1)} \leq-D_{3}\left(y^{2}+z^{2}+w^{2}\right)+\frac{1}{\eta}\left(\gamma_{1}(t)+\gamma_{2}(t)\right) V+(\beta y+z+\alpha w) e(t) .
$$


By condition $\left(H_{1}\right)$ and using the inequalities $(2.7),(2.8),(2.9)$ together with, $2 u v \leq u^{2}+v^{2}$, we obtain

$$
\begin{aligned}
& \mathrm{W}_{(2.1)}=\left(. V_{(? ?)}-\frac{1}{\eta}\left(\gamma_{1}(t)+\gamma_{2}(t)\right) V\right) e^{-\frac{1}{\eta} \int_{0}^{t}\left(\gamma_{1}(s)+\gamma_{2}(s)\right) d s} \\
& \leq\left(-D_{3}\left(y^{2}+z^{2}+w^{2}\right)+(\beta y+z+\alpha w) e(t)\right) e^{-\frac{1}{\eta} \int_{0}^{t}\left(\gamma_{1}(s)+\gamma_{2}(s)\right) d s}
\end{aligned}
$$

$$
\leq D_{4}(|y|+|z|+|w|)|e(t)|
$$

$\leq D_{4}\left(3+y^{2}+z^{2}+w^{2}\right)|e(t)|$

$\leq D_{4}\left(3+\frac{1}{D_{2}} W\right)|e(t)|$

$\leq 3 D_{4}|e(t)|+\frac{D_{4}}{D_{2}} W|e(t)|$,

where $D_{4}=\max \{\alpha, \beta, 1\}$. Integrating (2) from 0 to $t$, using the condition $\left(H_{3}\right)$ and the Gronwall inequality, we have

$\mathrm{W}(\mathrm{t}, \mathrm{x}, \mathrm{y}, \mathrm{z}, \mathrm{w}) \leq W(0, x(0), y(0), z(0), w(0))+3 D_{4} \eta_{3}$

$+\frac{D_{4}}{D_{2}} \int_{0}^{t} W(s, x(s), y(s), z(s), w(s))|e(s)| d s$

$\leq\left(W(0, x(0), y(0), z(0), w(0))+3 D_{4} \eta_{3}\right) e^{\frac{D_{4}}{D_{2}} \int_{0}^{t}|e(s)| d s}$

$\leq\left(W(0, x(0), y(0), z(0), w(0))+3 D_{4} \eta_{3}\right) e^{\frac{D_{4}}{D_{2}} \eta_{3}}=\lambda_{1}<\infty$.

In view of inequalities (2.8) and (2.12), we get

$$
\left(x^{2}+y^{2}+z^{2}+w^{2}\right) \leq \frac{1}{D_{2}} W \leq \lambda_{2}
$$

where $\lambda_{2}=\frac{\lambda_{1}}{D_{2}}$. From the above inequality it follows that

$|x(t)| \leq \sqrt{\lambda_{2}},|y(t)| \leq \sqrt{\lambda_{2}},|z(t)| \leq \sqrt{\lambda_{2}},|w(t)| \leq \sqrt{\lambda_{2}} \quad$ for all $t \geq 0$. 
By using (2.13) and the condition (ii) and since $x^{\prime}=y$ and $x^{\prime \prime}=\frac{1}{g(x)} z$ we obtain

$|x(t)| \leq \sqrt{\lambda_{2}},\left|x^{\prime}(t)\right| \leq \sqrt{\lambda_{2}},\left|x^{\prime \prime}(t)\right|=\left|\frac{1}{g(x(t))} z(t)\right| \leq \frac{1}{m} \sqrt{\lambda_{2}}$ for all $t \geq 0$.

On the other hand, conditions (ii), $\left(H_{4}\right)$ and $(2.14)$, show that

$$
\left|\theta_{1}(t)\right|=\left|\frac{g^{\prime}(x(t))}{g^{2}(x(t))} x^{\prime}(t)\right|<\frac{\eta_{4}}{m^{2}} \sqrt{\lambda_{2}} \quad \text { for all } t \geq 0
$$

According to $(2.13),(2.15)$ and since $x^{\prime \prime \prime}(t)=\frac{1}{g(x(t))} w(t)-\theta_{1}(t) z(t)$, we get

$$
\left|x^{\prime \prime \prime}(t)\right| \leq \frac{1}{g(x(t))}|w(t)|+\left|\theta_{1}(t)\right||z(t)| \leq \frac{1}{m} \sqrt{\lambda_{2}}+\frac{\eta_{4}}{m^{2}} \lambda_{2} \quad \text { for all } t \geq 0 .
$$

Square integrable solutions: Now we proof the square integrability of solutions and their derivatives. let $\rho>0$, we define $F_{t}=F(t, x(t), y(t), z(t), w(t))$ as

$$
F_{t}=W+\rho \int_{0}^{t}\left(y^{2}(s)+z^{2}(s)+w^{2}(s)\right) d s
$$

where $W=W(t, x, y, z)$ is is defined as (2.2). Note that $F_{t}$ is positive definite since $W$ is positive definite. From (2.10), and the estimate

$$
e^{-\frac{1}{\eta}\left(\eta_{1}+\frac{\eta_{2}}{m^{2}}\right)} \leq e^{-\frac{1}{\eta} \int_{0}^{t}\left(\gamma_{1}(s)+\gamma_{2}(s)\right) d s} \leq 1
$$

we obtain

$$
. F_{t(2.1)} \leq-D_{3}\left(y^{2}(t)+z^{2}(t)+w^{2}(t)\right) e^{-\frac{1}{\eta}\left(\eta_{1}+\frac{\eta_{2}}{m^{2}}\right)}
$$




$$
\begin{gathered}
+D_{4}(|y(t)|+|z(t)|+|w(t)|)|e(t)| \\
+\rho\left(y^{2}(t)+z^{2}(t)+w^{2}(t)\right) .
\end{gathered}
$$

Choosing $\rho=D_{3} e^{-\frac{1}{\eta}\left(\eta_{1}+\frac{\eta_{2}}{m^{2}}\right)}$ we get

$$
\begin{gathered}
. F_{t(? ?)} \leq D_{4}\left(3+\frac{1}{D_{2}} W\right)|e(t)| \\
\leq 3 D_{4}|e(t)|+\frac{D_{4}}{D_{2}} F_{t}|e(t)| .
\end{gathered}
$$

Integrating (2.18) from 0 to $t$, using the condition $\left(H_{3}\right)$ and the Gronwall inequality, we have

$$
\begin{aligned}
& \mathrm{F}_{t} \leq F_{0}+3 D_{4} \eta_{3}+\frac{D_{4}}{D_{2}} \int_{0}^{t} F_{s}|e(s)| d s \\
\leq & \left(F_{0}+3 D_{4} \eta_{3}\right) e^{\frac{D_{4}}{D_{2}} \int_{0}^{t}|e(s)| d s} \\
\leq & \left(F_{0}+3 D_{4} \eta_{3}\right) e^{\frac{D_{4}}{D_{2}} \eta_{3}}=\lambda_{3}<\infty .
\end{aligned}
$$

from the above it follows that,

$$
\lim _{t \rightarrow \infty} F_{t} \leq \lambda_{3}
$$

hence

$$
\int_{0}^{\infty} y^{2}(s) d s<\lambda_{3} \quad, \quad \int_{0}^{\infty} z^{2}(s)<\lambda_{3} \text { and } \int_{0}^{\infty} w^{2}(s) d s<\lambda_{3} .
$$

By using (2.20) and the condition (ii) and since $x^{\prime}=y$ and $x^{\prime \prime}=\frac{1}{g(x)} z$ we obtain

$(2.21) \int_{0}^{\infty} x^{\prime 2}(s) d s \leq \lambda_{3} \quad, \quad \int_{0}^{\infty} x^{\prime \prime 2}(s) d s \leq \frac{1}{m} \int_{0}^{\infty} z^{2}(s) d s \leq \frac{\lambda_{3}}{m}=\lambda_{4}$.

On the other hand, Combining the conditions (ii), $\left(H_{4}\right)$ and (??), (2.14), gives 


$$
\theta_{1}^{2}(t)=\left[\frac{g^{\prime}(x(t))}{g^{2}(x(t))} x^{\prime}(t)\right]^{2} \leq \frac{\eta_{4}^{2}}{m^{4}} \lambda_{3}, \quad \text { for all } t \geq 0
$$

Using again inequality $2 u v \leq u^{2}+v^{2}$ we get

$$
\begin{aligned}
& \int_{0}^{\infty} \quad x^{\prime \prime \prime 2}(s) d s=\int_{0}^{\infty} \frac{w^{2}(s)}{g^{2}(x(s))} d s+\int_{0}^{\infty} \theta_{1}^{2}(s) z^{2}(s) d s-2 \int_{0}^{\infty} \frac{\theta_{1}(s)}{g(x(s))} z(s) w(s) d s \\
& \quad \leq 2 \frac{w^{2}(s)}{g^{2}(x(s))} d s+2 \int_{0}^{\infty} \theta_{1}^{2}(s) z^{2}(s) d s \\
& \quad \leq 2 N \int_{0}^{\infty}\left(z^{2}(s)+w^{2}(s)\right) d s \leq 4 N \lambda_{3}=\lambda_{5},
\end{aligned}
$$

where $N=\frac{1}{m^{2}} \max \left\{1, \frac{\eta_{4}^{2}}{m^{2}} \lambda_{3}\right\}$. Next, multiply (??) by $x(t)$ and integrate by parts from 0 to $t$ all the terms on the LHS of (??) we obtain

where

$$
\mathrm{I}_{1}(t)=-\left(g^{\prime}(x(t)) x^{\prime}(t) x^{\prime \prime}(t)+g(x(t)) x^{\prime \prime \prime}(t)\right) x(t)+g(x(t)) x^{\prime \prime}(t) x^{\prime}(t)-
$$

$\int_{0}^{t} g(x(s)) x^{\prime \prime 2}(s) d s$,

$I_{2}(t)=-a(t) p(x(t)) x(t) x^{\prime \prime}(t)+\int_{0}^{t} a^{\prime}(s) p(x(s)) x(s) x^{\prime \prime}(s) d s+\int_{0}^{t} a(s) p(x(s)) x^{\prime}(s) x^{\prime \prime}(s) d s$, $I_{3}(t)=-b(t) q(x(t)) x(t) x^{\prime}(t)+\int_{0}^{t} b^{\prime}(s) q(x(s)) x(s) x^{\prime}(s) d s+\int_{0}^{t} b(s) q(x(s)) x^{\prime 2}(s) d s$, $I_{4}(t)=-\frac{1}{2} c(t) f(x(t)) x^{2}(t)+\frac{1}{2} \int_{0}^{t} c^{\prime}(s) f(x(s)) x^{2}(s) d s+\frac{1}{2} \int_{0}^{t} c(s) f^{\prime}(x(s)) x^{\prime}(s) x^{2}(s) d s$, $I_{5}(t)=\int_{0}^{t} e(s) x(s) d s$,

and

$$
\begin{aligned}
R_{0}= & \left(g^{\prime}(x(0)) x^{\prime}(0) x^{\prime \prime}(0)+g(x(0)) x^{\prime \prime \prime}(0)\right) x(0) \\
& -g(x(0)) x^{\prime \prime}(0) x^{\prime}(0)+a(0) p(x(0)) x(0) x^{\prime \prime}(0) \\
& +b(0) q(x(0)) x(0) x^{\prime}(0)+\frac{1}{2} c(0) f(x(0)) x^{2}(0) .
\end{aligned}
$$


From $(2.14),(2.16),(2.21)$ and the conditions (i), (ii), $\left(H_{1}\right) \sim\left(H_{4}\right)$, we have

and

$$
\begin{aligned}
I_{1}(t) & \leq\left(\frac{\eta_{4}}{m} \lambda_{2}^{\frac{3}{2}}+M\left(\frac{1}{m} \sqrt{\lambda_{2}}+\frac{\eta_{4}}{m^{2}} \lambda_{2}\right)\right) \sqrt{\lambda_{2}}+\frac{M}{m} \lambda_{2}+M \int_{0}^{t} x^{\prime \prime 2}(s) d s \\
I_{2}(t) & \leq a_{1} \frac{M}{m} \lambda_{2}+\frac{M}{m} \lambda_{2} \int_{0}^{t}\left|a^{\prime}(s)\right| d s+a_{1} M \int_{0}^{t} x^{\prime}(s) x^{\prime \prime}(s) d s \\
& \leq a_{1} \frac{M}{m} \lambda_{2}+\frac{1}{2} a_{1} M \lambda_{2}+\frac{M}{m} \lambda_{2} \int_{0}^{t}\left|a^{\prime}(s)\right| d s \\
I_{3}(t) & \leq b_{1} q_{1} \lambda_{2}+q_{1} \lambda_{2} \int_{0}^{t}\left|b^{\prime}(s)\right| d s+b_{1} q_{1} \int_{0}^{t} x^{\prime 2}(s) d s \\
I_{4}(t) & \leq \frac{1}{2} c_{1} M \lambda_{2}+\frac{1}{2} M \lambda_{2} \int_{0}^{t}\left|c^{\prime}(s)\right| d s,+\frac{1}{2} c_{1} \lambda_{2}^{\frac{3}{2}} \int_{0}^{t}\left|f^{\prime}(s)\right| d s \\
I_{5}(t) & \leq \sqrt{\lambda_{2}} \int_{0}^{t}|e(s)| d s .
\end{aligned}
$$

Hence

$$
\begin{aligned}
& \lim _{t \rightarrow+\infty} I_{1}(t) \leq\left(\frac{\eta_{4}}{m} \lambda_{2}^{\frac{3}{2}}+M\left(\frac{1}{m} \sqrt{\lambda_{2}}+\frac{\eta_{4}}{m^{2}} \lambda_{2}\right)\right) \sqrt{\lambda_{2}}+\frac{M}{m} \lambda_{2}+M \lambda_{4}=R_{1}, \\
& \lim _{t \rightarrow+\infty} I_{2}(t) \leq a_{1} \frac{M}{m} \lambda_{2}+\frac{1}{2} a_{1} M \lambda_{2}+\frac{M}{m} \lambda_{2} \eta_{1}=R_{2}, \\
& \lim _{t \rightarrow+\infty} I_{3}(t) \leq b_{1} q_{1} \lambda_{2}+q_{1} \lambda_{2} \eta_{1}+b_{1} q_{1} \lambda_{3}=R_{3}, \\
& \lim _{t \rightarrow+\infty} I_{4}(t) \leq \frac{1}{2} c_{1} M \lambda_{2}+\frac{1}{2} M \lambda_{2} \eta_{1}+\frac{1}{2} c_{1} \lambda_{2}^{\frac{3}{2}} \eta_{2}=R_{4}, \text { and } \lim _{t \rightarrow+\infty} I_{5}(t) \leq \sqrt{\lambda_{2}} \eta_{3}=R_{5} .
\end{aligned}
$$

Thus

$$
\lim _{t \rightarrow+\infty}\left(I_{1}(t)+I_{2}(t)+I_{3}(t)+I_{4}(t)+I_{5}(t)\right) \leq \sum_{i=1}^{5} R_{i}<\infty .
$$

Consequently, (2.23), (2.24) and condition (iii) gives

$$
\int_{0}^{\infty} x^{2}(s) d s \leq \frac{1}{d_{0} \delta} \int_{0}^{\infty} d(s) x(s) h(x(s)) d s \leq \frac{1}{d_{0} \delta} \sum_{i=0}^{5} R_{i}<\infty .
$$

The proof of the theorem is now completed.

If $e(t)=0$, similarly to above proof, the inequality (2.10) becomes

$$
. W_{(? ?)}=\left(. V_{(? ?)}-\frac{1}{\eta}\left(\gamma_{1}(t)+\gamma_{2}(t)\right) V\right) e^{-\frac{1}{\eta} \int_{0}^{t}\left(\gamma_{1}(s)+\gamma_{2}(s)\right) d s}
$$




$$
\begin{aligned}
& \leq-D_{3}\left(y^{2}+z^{2}+w^{2}\right) e^{-\frac{1}{\eta} \int_{0}^{t}\left(\gamma_{1}(s)+\gamma_{2}(s)\right) d s} \\
& \leq-\mu\left(y^{2}+z^{2}+w^{2}\right),
\end{aligned}
$$

where $\mu=D_{3} e^{-\frac{1}{\eta}\left(\eta_{1}+\frac{\eta_{2}}{m^{2}}\right)}$. It is easy to see that the only solution of system (2.1) for which $. W_{(? ?)}(t, x, y, z, w)=0$ is the solution $x=y=z=w=0$. Hence the trivial solution of equation (1.5) is uniformly asymptotically stable, and the same conclusion as in the proof of Theorem 3.2 can be drawn for the square integrability of solutions of equation (1.5).

\section{Example}

We consider the following fourth order non-autonomous differential equation

$$
\begin{aligned}
& \left(\left(\frac{x^{2} \sin x+5 x^{4}+5}{5\left(1+x^{4}\right)}\right) x^{\prime \prime}\right)^{\prime \prime}+\left(e^{-t} \sin t+2\right)\left(\left(\frac{x+4 e^{x}+4 e^{-x}}{4\left(e^{x}+e^{-x}\right)}\right) x^{\prime \prime}\right)^{\prime} \\
& \quad+\left(\frac{\cos t+7 t^{2}+7}{1+t^{2}}\right)\left(\left(\frac{\sin x+6 e^{x}+6 e^{-x}}{e^{x}+e^{-x}}\right) x^{\prime}\right)^{\prime} \\
& \quad+\left(e^{-2 t} \sin ^{3} t+2\right)\left(\frac{x \cos x+5 x^{4}+5}{5\left(1+x^{4}\right)}\right) x^{\prime}+\left(\frac{\cos ^{2} t+t^{2}+1}{10\left(1+t^{2}\right)}\right)\left(\frac{x}{x^{2}+1}\right)=\frac{2 \sin t}{t^{2}+1} .(3 .
\end{aligned}
$$

Taking

$g(x)=\frac{x^{2} \sin x+5 x^{4}+5}{5\left(1+x^{4}\right)}, p(x)=\frac{x+4 e^{x}+4 e^{-x}}{4\left(e^{x}+e^{-x}\right)}, q(x)=\frac{\sin x+6 e^{x}+6 e^{-x}}{e^{x}+e^{-x}}$, $f(x)=\frac{x \cos x+5 x^{4}+5}{5\left(1+x^{4}\right)}, h(x)=\frac{x}{x^{2}+1}, a(t)=e^{-t} \sin t+2, b(t)=$ $\frac{\cos t+7 t^{2}+7}{1+t^{2}}$ $c(t)=e^{-2 t} \sin ^{3} t+2, d(t)=\frac{\cos ^{2} t+t^{2}+1}{10\left(1+t^{2}\right)} \quad$ and $\quad e(t)=\frac{2 \sin t}{t^{2}+1}$.

we have

$m=\frac{9}{10}, M=\frac{11}{10}, q_{0}=\frac{11}{2}, q_{1}=\frac{13}{2}, h_{0}=\frac{5}{2}, \delta_{0}=\frac{5}{3}, a_{0}=$ 
$1, a_{1}=3, b_{0}=6$,

$b_{1}=8, c_{0}=1, c_{1}=3, d_{0}=\frac{1}{10}, d_{1}=\frac{1}{5}$,

we find

$$
\frac{h_{0}}{m}-\frac{a_{0} m \delta_{0}}{d_{1}}=-4.55 \leq h^{\prime}(x) \leq 1.1 \leq \frac{h_{0}}{2 M},
$$

and

$$
\begin{aligned}
\kappa_{1} & =\frac{a_{1} h_{0} d_{1} M^{2}}{c_{0} m^{3}}+\frac{M^{3}\left(c_{1}+\delta_{0}\right)}{a_{0} m^{2}}+a_{0} a_{1} m(M-1) \leq 10 \\
\kappa_{2} & =\frac{2 d_{1} h_{0} a_{0}}{c_{0}(M-1)}\left(\frac{1}{m}-\frac{1}{M}\right)^{2}+2 \frac{c_{0} M}{a_{0}}+2 a_{1} \frac{d_{1} h_{0} M}{c_{0} m^{3}}+\frac{c_{0} c_{1}\left(M^{2}+2\right) m M}{d_{1} h_{0}} \leq 27
\end{aligned}
$$

Also

$$
\begin{aligned}
\int_{-\infty}^{+\infty}\left|g^{\prime}(x)\right| d x= & \frac{1}{5} \int_{-\infty}^{+\infty}\left|\frac{-4 x^{5} \sin x+\left(2 x \sin x+x^{2} \cos x\right)\left(x^{4}+1\right)}{\left(x^{4}+1\right)^{2}}\right| d x \\
\leq & \frac{1}{5} \int_{-\infty}^{+\infty}\left(\frac{x^{2}}{x^{4}+1}+\frac{4 x^{6}}{\left(x^{4}+1\right)^{2}}+\frac{2 x^{2}}{x^{4}+1}\right) d x=\frac{3}{5} \sqrt{2} \pi \\
\int_{-\infty}^{+\infty}\left|p^{\prime}(x)\right| d x= & \frac{1}{4} \int_{-\infty}^{+\infty}\left|\frac{1}{e^{x}+e^{-x}}+x \frac{e^{-x}-e^{x}}{\left(e^{x}+e^{-x}\right)^{2}}\right| d x \\
\leq & \frac{1}{4} \int_{-\infty}^{0}\left(\frac{1}{\left.e^{x}+e^{-x}-x \frac{e^{-x}-e^{x}}{\left(e^{x}+e^{-x}\right)^{2}}\right) d x}\right. \\
& +\frac{1}{4} \int_{0}^{+\infty}\left(\frac{1}{e^{x}+e^{-x}}-x \frac{e^{-x}-e^{x}}{\left(e^{x}+e^{-x}\right)^{2}}\right) d x=\frac{\pi}{4} \\
\int_{-\infty}^{+\infty}\left|q^{\prime}(x)\right| d x= & \frac{1}{5} \int_{-\infty}^{+\infty}\left|\frac{\left(e^{x}+e^{-x}\right) \cos x-\left(e^{x}-e^{-x}\right) \sin x}{\left(e^{x}+e^{-x}\right)^{2}}\right| d x \\
\leq & \frac{1}{5} \int_{-\infty}^{+\infty}\left(\frac{1}{e^{x}+e^{-x}}+\frac{x}{\left(e^{x}+e^{-x}\right)^{2}}\left(e^{x}-e^{-x}\right)\right) d x=\frac{1}{5} \pi \\
\int_{-\infty}^{+\infty}\left|f^{\prime}(x)\right| d x \leq & \frac{1}{5} \int_{-\infty}^{+\infty}\left|\frac{(\cos x-x \sin x)\left(x^{4}+1\right)-4 x^{4} \cos x}{\left(x^{4}+1\right)^{2}}\right| d x
\end{aligned}
$$




$$
\begin{aligned}
& =\frac{1}{5} \int_{-\infty}^{+\infty}\left|\frac{\cos x}{x^{4}+1}-4 x^{4} \frac{\cos x}{\left(x^{4}+1\right)^{2}}-x \frac{\sin x}{x^{4}+1}\right| d x \\
& \leq \frac{1}{5} \int_{-\infty}^{+\infty}\left(\frac{5}{x^{4}+1}+\frac{x^{2}}{x^{4}+1}\right) d x=\frac{9}{10} \sqrt{2} \pi .
\end{aligned}
$$

Then

$$
\int_{-\infty}^{+\infty}\left(\left|g^{\prime}(s)\right|+\left|p^{\prime}(s)\right|+\left|q^{\prime}(s)\right|+\left|f^{\prime}(s)\right|\right) d s<\infty, \quad\left|g^{\prime}(t)\right|<3,
$$

and

$$
\begin{aligned}
& \int_{0}^{+\infty}|e(t)| d t=\int_{0}^{+\infty}\left|\frac{2 \sin t}{t^{2}+1}\right| d t \leq \int_{0}^{+\infty} \frac{2}{t^{2}+1} d t=\pi, \\
& \int_{0}^{+\infty}\left|a^{\prime}(t)\right| d t=\int_{0}^{+\infty}\left|(\cos t) e^{-t}-(\sin t) e^{-t}\right| d x \leq \int_{0}^{+\infty} 2 e^{-t} d x=2, \\
& \int_{0}^{+\infty}\left|b^{\prime}(t)\right| d t=\int_{0}^{+\infty}\left|-\frac{\sin t}{t^{2}+1}-2 t \frac{\cos t}{\left(t^{2}+1\right)^{2}}\right| d x \leq \int_{0}^{+\infty}\left(\frac{1}{t^{2}+1}+\frac{2|t|}{\left(t^{2}+1\right)^{2}}\right) d x \\
& \quad \leq \int_{0}^{+\infty}\left(\frac{1}{t^{2}+1}+\frac{t^{2}+1}{\left(t^{2}+1\right)^{2}}\right) d x=\int_{0}^{+\infty} \frac{2}{t^{2}+1} d x=\pi, \\
& \quad \int_{0}^{+\infty}\left|c^{\prime}(t)\right| d t=\int_{0}^{+\infty}\left|c^{\prime}(t)\right| d x=\int_{0}^{+\infty}\left|3\left(\cos t \sin ^{2} t\right) e^{-2 t}-2\left(\sin ^{3} t\right) e^{-2 t}\right| d x \\
& \leq \int_{0}^{+\infty} 5 e^{-2 t} d x=\frac{5}{2}, \\
& \quad \int_{0}^{+\infty}\left|d^{\prime}(t)\right| d t=\int_{0}^{+\infty}\left|-2(\cos t) \frac{\sin t}{t^{2}+1}-2 t \frac{\cos ^{2} t}{\left(t^{2}+1\right)^{2}}\right| d x \\
& \leq \int_{0}^{+\infty}\left(\frac{2}{t^{2}+1}+\frac{2|t|}{\left(t^{2}+1\right)^{2}}\right) d x \leq \int_{0}^{+\infty} \frac{3}{t^{2}+1} d x=\frac{3 \pi}{2} .
\end{aligned}
$$

Then

$$
\int_{0}^{+\infty}\left(\left|a^{\prime}(t)\right|+\left|b^{\prime}(t)\right|+\left|c^{\prime}(t)\right|+\left|d^{\prime}(t)\right|\right) d t<+\infty .
$$

Thus all the assumptions of Theorem (2) hold, this shows that every solution $x(t)$ of (??) and their derivatives $x^{\prime}(t), x^{\prime \prime}(t)$ and $x^{\prime \prime \prime}(t)$ are bounded and square integrable.

Acknowledgement The author of the paper would like to express sincere thanks to the anonymous referees for their invaluable corrections and suggestions. 


\section{References}

[1] Afuwape, A .U. and Adesina, O. A.; Frequency-domain approach to stability and periodic solutions of certain fourth-order non-linear differential equations. Nonlinear Stud. 12, No. 3, pp. 259-269, (2005).

[2] Andres, J., and Vlček, V.; On the existence of square integrable solutions and their derivatives to fourth and fifth order differential equations. Acta Universitatis Palackianae Olomucensis. Facultas Rerum Naturalium. Mathematica, Vol. 28, No. 1, pp. 65-86, (1989).

[3] Burton T. A., Stability and periodic solutions of ordinary and functional differential equations .Mathematics in science and engineering, Volume 178, Academic Press, Inc, (1985).

[4] Burton T.A., Volterra Integraln and Differential Equations, Mathematics in Science and Engineering Vol. (202), 2nd edition, (2005).

[5] Chin, P. S. M.; Stability results for the solutions of certain fourth-order autonomous differential equations. Internat. J. Control. 49, No. 4, pp. 1163-1173, (1989).

[6] Ezeilo, J. O. C.; On the Boundedness and the Stability of Solution of some Fourth Order Equations, J. Math. Anal. Appl. 5, pp. 136-146, (1962).

[7] Ezeilo, J. O. C.; A Stability result for Solutions of a Certain Fourth Order Diffrential Equations. J.London Math. Soc. 37, pp. 28-32, (1962).

[8] Harrow, M.; A Stability result for Solutions of a Certain Fourth Order Homogeneous Differential Equations, J. London Math. Soc. 42, pp. 51-56, (1967).

[9] Harrow, M.; On the Boundedness and the Stability of Solutions of some Differential Equations of the Fourth Order, SIAM, J. Math. Anal. 1, pp. 27-32, (1970).

[10] Hara, T. On the asymptotic behavior of the solutions of some third and fourth order non-autonomous differential equations. Publ. RIMS, Kyoto Univ. 9, pp. 649-673, (1974).

[11] Hara, T.; On the asymptotic behavior of solutions of some third order ordinary differential equations. Proc. Japan Acad., 47 (1971).

[12] Omeike, M. O.; Boundedness of solutions of the fourth order differential equation with oscillatory restoring and forcing terms. An. Ştiint . Univ. Al. I. Cuza Iaşi. Mat. (N.S.) 54, No. 1, pp. 187-195, (2008). 
[13] Remili, M. and Oudjedi, D. L.; Uniform stability and boundedness of a kind of third order delay differential equations. Bull. Comput. Appl. Math., Vol 2, No. 1, (2014).

[14] Remili, M. and Oudjedi, D. L.; Stability and boundedness of the solutions of non autonomous third order differential equations with delay. Acta Univ. Palack. Olomuc. Fac. Rerum Natur. Math. 53, No. 2, pp. 139-147, (2014).

[15] Remili, M. and Beldjerd, D.; On the asymptotic behavior of the solutions of third order delay differential equations. Rend. Circ. Mat. Palermo, Vol 63, No $3,(2014)$.

[16] Shair, A.; Asymptotic properties of linear fourth order differential equations. American mathematical society volume 59. No 1, august (1976).

[17] Tiryaki,A. and Tunc,C.; Boundedness and the Stability Properties of Solutions of Certain Fourth Order Differential Equations via the Intrinsic Method, Analysis, 16, pp. 325-334, (1996).

[18] Tunç,C.; A Note on the Stability and Boundedness Results of Certain Fourth Order Differential Equations, Applied Mathematics and Computation, 155, No. 3, pp. 837-843, (2004).

[19] Tunç,C.; Some Stability and Boundedness Results for the Solutions of Certain Fourth Order Differential Equations, Acta Univ. Palacki Olomouc. Fac. Rerum Natur. Math. 44, pp. 161-171, (2005).

[20] Tunç, C.; An ultimate Boundedness Result for a Certain System of Fourth Order Nonlinear Differential Equations, Differential Equations and Applications, Vol. 5, pp. 16-174, (2005).

[21] Tunç,C. and Tiryaki,A.; On the Boundedness and the Stability Results for the Solutions of Certain Fourth Order Differential Equations via the Intrinsic Method, Applied Mathematics and Mechanics, 17, No. 11, pp. 1039-1049, (1996).

[22] Tiryaki. A.; Tunç, C. Constructing Lyapunov functions for certain fourthorder autonomous differential equations. Indian J. Pure Appl. Math. 26, No. 3, pp. 225-232, (1995).

[23] Tunç, C.; Stability and boundedness of solutions to certain fourth order differential equations. Electronic Journal of Differential Equations, Vol. 2006, No. 35, pp. 1-10, (2006).

[24] Tunç, C.; Some remarks on the stability and boundedness of solutions of certain differential equations of fourth-order. and Computational and Applied Mathematics, Volume 26, N. 1, pp. 1-17, (2007). 
[25] Tunç, C., and Ates, M.; Boundedness of Solutions to Differential Equations of Fourth Order with Oscillatory Restoring and Forcing Terms. Discrete Dynamics in Nature and Society Volume, pp. 1-6, (2013).

[26] Vlček, V.; On the boundedness of solutions of a certain fourth-order nonlinear differential equation. Acta Universitatis Palackianae Olomucensis. Facultas Rerum Naturalium. Mathematica, Vol. 27, No. 1, pp. 273-288, (1988).

[27] Yoshizawa, T.; Stability Theory by Liapunovs Second Method, The Mathematical Society of Japan, Tokyo, (1966).

\section{Moussadek Remili}

Department of Mathematics

University of Oran 1 Ahmed Ben Bella

31000 Oran

Algeria

e-mail : remilimous@gmail.com

and

\section{Mebrouk Rahmane}

Department of Mathematics

University of Oran 1 Ahmed Ben Bella

31000 Oran

Algeria

e-mail : mebroukrahmane@yahoo.fr 